\title{
CONTENIDO Y CONCIENCIA: \\ EL DEBATE EN TORNO A LOS QUALLA
}

ELEONORA ORLANDO

DEPARTAMENTO DE FILOSOFÍA

UNIVERSIDAD DE BUENOS AIRES

El tema de este artículo es la polémica en torno a los qualia, es decir, en torno a la existencia de un aspecto del contenido de los estados mentales que excede lo puramente intencional. La introducción contiene un estado de la cuestión acerca del debate y su lugar en el panorama actual de la filosofía de la mente. A continuación, reconstruyo y evalúo los argumentos más importantes ofrecidos en favor de la existencia de los qualia. Mi diagnóstico es que todos ellos presentan un mismo problema, derivado de lo que denomino "la estrategia epistémica". La sección siguiente está centrada en el examen de los argumentos del Espectro Invertido y la Tierra Invertida, los cuales son presentados como ejemplos de argumentos basados en la concebibilidad. Nuevamente, la tesis propuesta es que no prueban lo que se supone que deben probar, a saber, que los qualia existen. Finalmente, concluyo que la existencia de los qualia, tal como han sido tradicionalmente concebidos, no puede fundamentarse en ningún argumento de estos tipos.

\section{Introducción}

"Qualia" es una palabra latina que se usa para designar ciertas propiedades de ciertos estados mentales. Más específicamente, se trata de propiedades fenomenológicas o cualitativas, es decir, propiedades constituidas por cómo es ("what it is like") tener esos estados. Pienso que la mejor manera de comprender la presunta naturaleza de los qualia es oponiéndolos a las propiedades intencionales o representacionales, es decir, a aquellas propiedades de los estados mentales constituidas por sus relaciones con objetos o propiedades del mundo. ${ }^{1}$ De modo que se supone que algunos estados mentales tienen dos tipos distintos de contenido: el contenido intencional o representacional, determinado por relaciones externas (o internas) y el

1 Esto presupone algún tipo de externalismo, pero podría ser fácilmente reformulado de modo tal que se adecue a una teoría internalista del contenido. 
contenido fenoménico o cualitativo, determinado por cómo es ("what it is like" o "how it feels") para un individuo mantener esas relaciones. Cabe aclarar que, en algunos casos, puede faltar el componente intencional, es decir, hay algunos estados mentales, como, por ejemplo, los dolores, que son puramente cualitativos.

Por otro lado, como señalé al comienzo, se considera que sólo ciertos estados mentales tienen contenido cualitativo, a saber, el subconjunto constituido por las percepciones (como las experiencias visuales y gustativas), las sensaciones corporales (como los dolores y las cosquillas), las imágenes (como las de las propias percepciones y acciones) y las cadenas de pensamientos (como cuando se piensa en imágenes o en palabras); en otros términos, lo que usualmente se designa mediante el término general "experiencia". ${ }^{2}$ Por el contrario, se suele considerar que las actitudes proposicionales tienen sólo contenido intencional —si bien no hay acuerdo absoluto al respecto. ${ }^{3}$

Para ser más específica acerca de la naturaleza de los qualia, es necesario tener en cuenta las propiedades - de segundo orden, puesto que son propiedades de propiedades- que lés son generalmente adscritas. Aun cuando tampoco hay acuerdo sobre este punto, se suele presuponer que los qualia son:

(i) intrínsecos: no relacionales, i.e., propiedades que un estado mental tiene en sí mismo y no en virtud de su relación con un objeto externo

2 Véase, por ejemplo, Lormand (1995b). Cabe aclarar que las cadenas de pensamientos no deben confundirse con actitudes proposicionales como las creencias: se trata del fenómeno, típicamente lento y serial, usualmente descrito como "hablar para sí mismo" o "pensar en palabras". Si bien tales pensamientos están asociados con creencias (y pueden ser causados por ellas), el contenido de unos y otras es claramente distinto. A modo de ejemplo, la creencia de que el pasto es verde representa el hecho de que el pasto es verde, pero el pensamiento asociado representa las palabras "El pasto es verde" o alguna imagen particular de una extensión verde de pasto.

3 Flanagan, por ejemplo, distingue un sentido estricto de un sentido amplio de "qualia". "Las creencias, pensamientos, esperanzas, expectativas y estados de actitud proposicional en general, así como las grandes estructuras narrativas, son cualitativas (o tienen componentes cualitativos) en este último sentido [amplio] [...] En nuestra fenomenología, las creencias, cuando son experimentadas, lo son de una manera distinta de los deseos, y éstos, a su vez, difieren de las expectativas" (Flanagan (1991), cap. 4, p. 67). La tesis parece tener más fundamento si se piensa, más que en las actitudes proposicionales, en los estados de ánimo. Según Lormand, sin embargo, estas teorías no hacen sino considerar como propios de las actitudes proposicionales a los qualia de los estados perceptuales concomitantes. En su opinión, si se hace abstracción de éstos últimos, desaparece todo aspecto cualitativo. Véase Lormand (1995 a y b). La tesis de Lormand tiene la ventaja de rescatar la idea intuitiva de que el carácter cualitativo de un estado mental está determinado por el modo en que éste es experimentado o sentido (en relación, por consiguiente, con una facultad de experimentar o percibir, que no parece en absoluto constitutiva del estado de tener una actitud proposicional). 
(ii) determinantes (en parte) de la representación externa

(iii) privados: no accesibles a la observación (pública) sino mediante la introspección

(iv) directos: accesibles a la conciencia de manera directa o no inferencial

(v) inefables: inexpresables en el lenguaje público e incomunicables

(vi) atómicos o simples: indivisibles.

Como puede preverse, la existencia de tales propiedades crearía un problema para el funcionalismo: si hay estados mentales con propiedades cualitativas así concebidas, entonces la tesis funcionalista de que los estados mentales son idénticos a estados funcionales parece ser, en principio, falsa. En otras palabras, si los qualia existen y poseen las características antes mencionadas, parece ser falso que el contenido mental pueda ser explicado en términos aceptables para el funcionalismo, es decir, en términos de las interacciones causales entre inputs, outputs y estados mentales, y en términos de las relaciones causales de éstos últimos con objetos del entorno. Dado que, según la caracterización anterior, los qualia son concebidos como propiedades intrínsecas de la experiencia, no parecen, en principio, ser reducibles a relaciones de ningún tipo. Por consiguiente, el compromiso ontológico con los qualia parece exigir un precio muy alto: la renuncia al funcionalismo como teoría general de la mente.

Por otro lado, si se opta por "quinear" los qualia -es decir, por negar resueltamente su existencia- ${ }^{4}$ es posible seguir siendo funcionalista pero se está amenazado por otro problema: la imposibilidad de distinguir claramente los estados mentales conscientes de los inconscientes. Los qualia parecen ofrecer el mejor modo de trazar la distinción en cuestión: son conscientes aquellos estados mentales que tienen contenido cualitativo o están acompañados por estados mentales con contenido cualitativo (percepciones, sensaciones corporales, imágenes y cadenas de pensamientos conscientes); son inconscientes aquellos estados mentales que no tienen contenido cualitativo ni están acompañados por estados mentales que lo tengan. Las teorías puramente funcionalistas de la mente, en cambio, tienen problemas a la hora de trazar la distinción. Por un lado, están las teorías metarrepresentacionales, según las cuales un estado mental es consciente en virtud de su relación funcional con otro estado que tiene la propiedad (intencional) de ser acerca de él. La conciencia requiere conciencia acerca de la conciencia, la cual, en una.teoría de gran influencia ha sido concebida como

4 La expresión está tomada de Dennett (1988). 
una conciencia proposicional o teórica (de que cierto estado mental existe). ${ }^{5}$ El problema es que no hay razón para pensar que nuestros pensamientos de orden superior (por ejemplo, nuestras creencias acerca de nuestros propios estados mentales) desaparecen durante el sueño; sin embargo, no es posible pensar que determinen estados mentales conscientes mientras uno duerme. Más aun, tales teorías involucran un regreso al infinito: un estado mental no puede ser consciente si nuestra representación del mismo es inconsciente; por consiguiente, es necesario que la metarrepresentación sea a su vez objeto de una (metameta) representación, y así sucesivamente. Finalmente, esta concepción parece excluir a los bebés y a los animales, quienes no tienen creencias acerca de sus propios estados mentales, del ámbito de la conciencia. La alternativa está representada por aquellas teorías que definen la conciencia en términos de las relaciones funcionales de un estado mental con otros, ninguno de los cuales constituye una representación acerca del primero. ${ }^{6}$ Se dice, por ejemplo, que son sus efectos sobre la acción y la memoria los que hacen a un estado mental consciente. El problema con este tipo de teorías es que no queda en absoluto claro cuáles son las relaciones funcionales que determinan la conciencia (y ciertamente, no parece verosímil sostener, como Dennett, que todas ellas lo hacen). ${ }^{7}$ En síntesis, si se quiere evitar el compromiso ontológico con los qualia, el desafío parece ser ofrecer una teoría de la mente puramente funcionalista que pueda dar cuenta de la diferencia entre conciencia e inconciencia -lo cual es, en principio, sumamente difícil. ${ }^{8}$

Frente a este dilema teórico -qualia vs. funcionalismo-, considero que el primer paso es examinar los argumentos más importantes que han sido esgrimidos en favor de la existencia de los qualia. Esto es lo que me propongo hacer en las próximas secciones.

\section{Los argumentos en favor de los qualia}

En mi opinión, los argumentos más interesantes y elaborados pueden ser clasificados en dos grupos: por un lado, el argumento epistemológico y su

5 Se trata de la teoría de Rosenthal, quien define la conciencia en términos de pensamientos (concebidos como actitudes proposicionales) de orden superior ("higher order thoughts"). Véase Rosenthal (1990).

6 Dennett (1991) y Rey (1992) y (1993).

7 Dennett sostiene que los supuestos qualia, determinantes del carácter consciente de una experiencia, no son sino "la suma total de todas las ideosincráticas disposiciones a actuar" que resultan de ella. Véase Dennett (1991), p. 387. Véase también la crítica de Lormand a Dennett en Lormand (1995a).

8 Ésta es la empresa en la que se ha embarcado, paradigmáticamente, Dennett. Véase Dennett (1991). 
pariente cercano, el argumento basado en la perspectiva; por otro, los argumentos basados en la concebibilidad y sus parientes cercanos, los basados en la posibilidad. ${ }^{9}$ El primer grupo se apoya en el carácter privado e inefable de ciertos aspectos mentales, mientras que el segundo lo hace en su carácter intrínseco o no relacional. En lo que sigue, explicaré en qué consiste cada uno de estos tipos de argumentos.

El argumento epistemológico fue propuesto por Jackson, por medio de varios ejemplos. ${ }^{10} \mathrm{~A}$ modo de ilustración, tomaré el ejemplo concerniente a María, una neurocientífica brillante, que ha pasado toda su vida encerrada en un cuarto blanco y negro y cuya sola relación con el mundo externo ha sido mediante un monitor blanco y negro, del cual ha aprendido todos los hechos físicos objetivos; en cierto momento, a María se le permite salir del cuarto, con lo que logra tener su primera experiencia visual de color. $\mathrm{El}$ punto de Jackson es que, al hacerlo, María aprende algo nuevo acerca de la visión del color, a saber, su carácter cualitativo; por consiguiente, se concluye que los qualia existen como aquellos aspectos de la experiencia que no pueden ser reducidos a hechos físicos de ningún tipo.

Un argumento similar a éste es el argumento basado en la perspectiva. La versión original fue presentada por Nagel como un argumento en contra del fisicalismo, pero bien puede ser dirigido contra el funcionalismo, usualmente considerado una versión del fisicalismo. ${ }^{11}$ El argumento de Nagel puede resumirse en las siguientes palabras: aun cuando llegáramos a conocer todos los hechos físicos objetivos acerca de los murciélagos (especialmente, el mecanismo preciso de su sistema de ecolocación), habrá siempre algo acerca de ellos que no sabremos porque no somos murciélagos. Desde la perspectiva del observador o de la tercera persona, hay un aspecto del murciélago que es inaccesible —el carácter particular de la experiencia "murcielágica" que sólo puede ser sentido desde la primera persona, es decir, siendo un murciélago. En otras palabras, desde el punto de vista científico, no es posible saber cómo es ("what it is like") ser un murciélago. Por consiguiente, una vez más, se concluye que los qualia existen como aquellos aspectos de la experiencia que se resisten a ser expresados en términos físicos - $-\mathrm{y}$, por tanto, funcionales.

Estos dos argumentos, si bien de un modo levemente distinto, enfatizan el mismo punto: como mencioné anteriormente, se trata fundamentalmente del carácter privado de los qualia, es decir, del hecho de que éstos no son

9 Además de los mencionados, pueden citarse el argumento a partir de la introspección y los argumentos que parten de la alucinación y otros casos de percepción anormal, como la visión borrosa y los "after-images".

10 Jackson (1982).

11 Nagel (1974). 
accesibles desde afuera (mediante la observación) sino sólo desde adentro (mediante la introspección). En el argumento de Jackson, es el tipo de acceso epistémico (qué tipo de conocimiento se tiene) lo que se considera que determina la perspectiva epistémica en cuestión: el conocimiento teórico o proposicional involucra esencialmente el punto de vista del observador. En el argumento de Nagel, es la ontología (quién o qué se es) lo que se considera que determina la mencionada perspectiva epistémica. En ambos casos, la idea es que la perspectiva del observador impide el acceso a ciertos aspectos de la mente. Pero todos los aspectos físicos de la mente pueden ser conocidos desde la perspectiva del observador. Se concluye entonces que los qualia existen como aquellos aspectos no físicos de la mente.

Se suele interpretar el argumento de Nagel como pretendiendo fundamentar la existencia de qualia comunes a una especie (como la de los murciélagos). El argumento de Jackson, en cambio, es neutral respecto de este punto: no es claro si María, al ver por primera vez algo rojo, aprende cómo es para un ser humano cualquiera o cómo es para ella en particular ver rojo. ${ }^{12}$ En otras palabras, no es claro si los límites de la privacidad son los límites individuales o los de la especie.

La Sexta meditación cartesiana proporciona un ejemplo del segundo grupa de argumentos, a saber, un argumento basado en la concebibilidad. Allí se dice que el cuerpo es tal que puede ser concebido como divisible en partes, pero la mente es tal que debe concebirse como indivisible; luego, la mente es distinta del cuerpo. ${ }^{13}$ Una versión aplicada a los qualia puede encontrarse en el tradicional argumento del Espectro Invertido. ${ }^{14}$ Según éste, un estado mental $M 1$ (en $t 1$ ) puede ser concebido como distinto de $M 2$ (en $t 2$ ) aun cuando no haya variación funcional alguna entre ambos; por consiguiente, los estados mentales son distintos de los estados funcionales. En otras palabras, hay un aspecto mental cualitativo que impide identificar los estados mentales con estados funcionales. Más recientemente, Block ha propuesto una nueva versión: el argumento de la Tierra Invertida. ${ }^{15}$ Este último deriva la misma conclusión de la premisa conversa: un estado mental $M 1$ (en $t 1$ ) puede ser concebido como idéntico a $M 2$ (en $t 2$ ) aun cuando haya variación funcional entre ambos. Los dos argumentos evidencian la estrategia de la concebibilidad: dado que podemos concebir un aspecto

12 A la luz de esta distinción, podría decirse que para Nagel uno puede conocer plenamente la sensación de rojo de otra persona mientras que para Jackson eso no sería posible puesto que las sensaciones de rojo son propias de cada individuo. Debo este comentario a un esclarecedor punto crítico del evaluador de Diánoia.

${ }^{13}$ Descartes (1641) (1982), Sexta meditación, pp. 178-179.

14 Este argumento puede encontrarse en muchos artículos de la literatura sobre qualia y conciencia; véase, por ejemplo, Lycan (1973) y Shoemaker (1982).

15 Block (1990). 
cualitativo invertido sin concebir un aspecto funcional invertido y podemos concebir un aspecto funcional invertido sin concebir un aspecto cualitativo invertido, los aspectos funcionales son distintos de los cualitativos.

Finalmente, quiero mencionar los argumentos basados en la posibilidad, en cuya versión interesante se reducen a un argumento basado en la concebibilidad: lo que es posible - o real en un mundo posible - no es sino lo que podemos concebir como real. Este tipo es ejemplificado por el argumento de Kripke en contra de la teoría de la identidad. ${ }^{16}$ Según Kripke, todos los enunciados de identidad entre nombres propios y términos generales verdaderos son (metafísicamente) necesarios, porque se considera que tales expresiones componentes denotan rígidamente ciertas entidades. ${ }^{17} \mathrm{Al}$ analizar tales enunciados, se encuentra una asimetría entre las identidades físicas (es decir, enunciados pertenecientes a las ciencias físicas) tales como

(1) el calor es el movimiento de las moléculas

y las identidades psicofísicas (es decir, enunciados pertenecientes a la psicología) tales como

(2) la sensación de rojo es la activación de los nervios $X$.

La asimetría reside en el hecho de que (1) es verdadera pero (2) es falsa, lo cual puede explicarse en los términos siguientes. La oración (1) es necesariamente verdadera puesto que el calor no puede existir sin el movimiento de las moléculas; cuando imaginamos un mundo donde parece haber calor sin movimiento de moléculas, lo que imaginamos no es realmente calor sino alguna otra sustancia con sus mismas características fenomenológicas (caliente, brillante, causante de incendio, etc.). Por lo tanto, podemos concluir que no puede haber calor real sin movimiento de moléculas, lo que permite fundamentar la necesidad de su identificación. Este razonamiento no puede extenderse a identidades como (2): cuando imaginamos un mundo donde parece haber una sensación de rojo sin que se activen los nervios $X$, no podemos pensar en ese mundo como un mundo donde existe algo con todas las características fenomenológicas de las sensaciones de rojo sin ser realmente sensaciones de rojo. La razón de ello es que algo con todas las características fenomenológicas de las sensaciones de rojo es justamente una sensación de rojo - y no puede ser ninguna otra cosa. En otras palabras, en el caso de las entidades psicológicas, la manera en que el fenómeno se nos aparece no puede ser distinguida del fenómeno mismo. Por consiguiente,

16 Kripke (1981), Tercera conferencia. Cabe señalar que la teoría de la identidad es un caso de fisicalismo tipo.

17. Un designador rígido es un término que denota a un mismo individuo o tipo en todos los mundos posibles. Véase Kripke (1981), Primera conferencia. 
estamos realmente concibiendo un mundo donde hay sensaciones de rojo sin que se activen los nervios $X$. La falta de simetría entre (1) y (2) puede asimismo ponerse de manifiesto apelando a la noción de "fijar la referencia de un término". Tanto en el caso de "calor" como en el de "sensación de rojo", la referencia se fija apelando a la manera en que el calor y el rojo son sentidos respectivamente. Sin embargo, en el primer caso, se trata de una propiedad accidental o contingente del calor; luego, al concebirse la posibilidad de que el calor no sea el movimiento de las moléculas, lo que se está concibiendo es, como se señaló anteriormente, la posibilidad de la existencia de algo que tiene las mismas propiedades fenomenológicas del calor pero que no es en realidad calor. En el segundo caso, en cambio, la manera en que el rojo es sentido es una propiedad esencial de la sensación de rojo; luego, no es posible separar la sensación de rojo de sus propiedades fenomenológicas y, por tanto, de concebir la existencia de algo que tenga las mismas propiedades fenomenológicas de las sensaciones de rojo sin ser en realidad una sensación de rojo. ${ }^{18}$ En esta medida, (2) no es necesariamente verdadera; por ende, dada la teoría kripkeana de la referencia, es falsa. Por consiguiente, todas las identidades psicofísicas como (2) son igualmente falsas; luego, la teoría de la identidad, que consiste en un conjunto de enunciados de ese tipo, es falsa.

Como puede preverse, el argumento de Kripke puede ser reformulado de modo que ataque el funcionalismo y defienda la existencia de los qualia. Supongamos que cierta teoría funcionalista de la mente implique un enunciado como

(3) tener una sensación de rojo es estar en el estado funcional $F$.

Recordemos que, en la visión de Kripke, si (3) es verdadera, es necesariamente verdadera, lo cual significa que no hay mundo posible en el que (3) sea falsa. Sin embargo, la situación en la cual un individuo está en $F \sin$ tener la sensación de rojo es perfectamente posible, a saber, es la situación en la que se encuentra, o podría encontrarse, un robot. Por lo tanto, (3), y todos los enunciados funcionalistas de identidad como (3), son falsos. Este argumento es conocido como el argumento de los Qualia Ausentes, y ha sido ofrecido por Block en varios artículos. ${ }^{19}$

Cabe destacar que, tanto en el caso de los argumentos del Espectro y la Tierra Invertidos como en el de los Qualia Ausentes, lo cualitativo queda identificado fundamentalmente no como un aspecto privado sino como un aspecto intrínseco (y, por tanto, no relacional) de la experiencia.

18 Debo la inserción de esta explicación al evaluador de Diánoia.

19 Véase, por ejemplo, Block (1978). Este artículo contiene el famoso ejemplo que involucra a la China. 
A continuación, me centraré en el análisis de lo que podría denominarse "la estrategia epistémica", la cual, si bien se encuentra paradigmáticamente ejemplificada por el argumento epistemológico, es común a todos los argumentos mencionados.

\section{Crítica de la estrategia epistémica}

1. El argumento epistemológico: las interpretaciones de re y de dicto

Antes que nada, creo que hay una formulación del argumento que constituye una petición de principio: en dicha versión, la existencia de los qualia, lejos de ser concluida, está presupuesta en las premisas. Considérese lo siguiente:

(1) María sabe todo acerca de los estados neuronales y funcionales.

(2) María no sabe nada acerca de los qualia.

Luego, (3) los estados neuronales y funcionales son distintos de los qualia.

La premisa (2) no parece constituir una descripción ontológicamente neutral del estado mental de María antes de salir del cuarto blanco y negro. Por consiguiente, es conveniente reformular el argumento de alguna otra manera, como, por ejemplo, la siguiente:

(1') María conoce todos los hechos físicos acerca de la visión del color.

(2') María no conoce nada acerca de la experiencia visual del color.

Luego, $\left(3^{\prime}\right)$ la experiencia visual del color es distinta de la visión física del color.

Luego, (4') los qualia existen en tanto aspectos no físicos de la experiencia visual del color.

En mi opinión, el problema de este argumento es el hecho de que intenta derivar una conclusión ontológica (acerca de lo que hay) a partir de premisas epistémicas (acerca de lo que un individuo conoce); en otras palabras, el argumento es inválido porque involucra lo que denomino "una estrategia epistémica". Esta estrategia se manifiesta en las dos lecturas principales (de re y de dicto) que pueden hacerse de él.

La interpretación de re. En primer lugar, el enemigo de los qualia puede objetar que las premisas son ambiguas: es posible considerar que la expresión "conocer" tiene un significado diferente en cada una de las premisas. Por consiguiente, bajo una interpretación de re de sus premisas, el argu- 
mento comete una falacia de equivocación. ${ }^{20}$ Esto se aprecia claramente en la siguiente reformulación:

(1") María conoce por descripción todos los hechos físicos acerca de la visión del color.

(2") María no conoce por familiaridad nada acerca de la visión del color (en otros términos, María no ha estado nunca familiarizada con el color).

Luego, $\left(3^{\prime \prime}\right)$ la familiaridad con el color (i.e., la experiencia visual del color) es distinta de todo hecho físico acerca de la visión del color.

Al eliminar la ambigüedad de las premisas, el argumento se muestra como un argumento claramente inválido y, en mi opinión, su invalidez descansa en la mencionada estrategia epistémica. María, al conocer por descripción todos los hechos físicos, podría saber todo lo que puede saberse acerca de la visión del color, i.e., podría tener el concepto pleno de visión del color, sin haber estado nunca familiarizada con el color, i.e., sin haber tenido nunca una experiencia visual del color. La experiencia, en tanto forma distinta de conocimiento, no añadiría un nuevo aspecto (no físico) sino un nuevo modo de acceder a los mismos hechos (la visión física del color). De este modo, la conclusión no se sigue: la experiencia visual podría ser un fenómeno enteramente físico.

Antes de explicar esto último con más detalle, haré algunas aclaraciones terminológicas. Considero que los conceptos y las sensaciones constituyen distintos tipos de representaciones mentales; en términos contemporáneos, puede decirse que los conceptos constituyen módulos más abstractos y cercanos al procesador central mientras que las sensaciones pertenecen almódulo perceptivo, relacionado tanto con los sentidos externos como con el sentido interno. (La convención elegida para designar tanto los conceptos como las sensaciones, es decir, las representaciones mentales en general son los corchetes comunes.) Cabe aclarar además que la disputa no es acerca de si las representaciones mentales, ya sean conceptos o sensaciones, son identificables con propiedades cualitativas sino acerca de si las representaciones mentales tienen o no propiedades cualitativas que constituyen parte de su contenido. (La convención elegida para designar contenidos son los corchetes angulares.) Ahora bien, es obvio que tener el concepto de, por ejemplo, una sensación de rojo y la capacidad requerida para aplicarlo adecuadamente - es decir, para distinguir una sensación de rojo de una sensación de

20. Véase Churchland (1985), pp. 23-24. 
verde- no implica tener una sensación de rojo. ${ }^{21}$ Pero, ¿implica esto que, al tenerla, la sensación agrega algo al concepto? Pienso que el problema del argumento epistemológico es que presupone una respuesta afirmativa a esta pregunta.

En mi opinión, la respuesta a la pregunta depende de la teoría de los conceptos que uno sostenga. Contrariamente a lo que el argumento presupone, es posible sostener que percibir cierto objeto o propiedad no agrega nada al concepto de ese objeto o propiedad -el cual pudo haberse adquirido independientemente de tal percepción (si bien no necesariamente independientemente de toda experiencia sensible). En otros casos, la percepción pudo haber sido la vía de adquisición del concepto al inicio de ese proceso. El punto es que puede pensarse que en ningún caso hay algo, más allá de cierta fuerza o nitidez, que la percepción aporte al concepto. En particular, no hay razón para creer que exista un componente del contenido de un concepto (en nuestro ejemplo, el de visión física de color) que sólo puede ser aportado por la experiencia (visual del color). En este punto, quisiera citar el siguiente comentario de Levin:

Lo que la determina [la contribución de la experiencia al conocimiento] no es el hecho de que la experiencia aporta un pedazo de conocimiento que no puede ser vislumbrado de ninguna otra manera sino el hecho de que lo hace tan eficientemente. La función de la experiencia aquí es primordialmente causal y evidencial...22

A modo de ejemplo, mi concepto de roble se formó sobre una base múltiple: un cuento que me contaron cuando tenía cinco años, una canción escuchada a los siete, un dibujo en el libro de lectura de tercer grado, una definición dada por mi maestra de quinto. Cuando tenía quince años, vi por primera vez un roble en el jardín de mi abuelo. La experiencia visual no agregó ningún rasgo nuevo respecto de lo que yo ya sabía acerca de los robles.

Podrá objetarse que un roble, a diferencia de una sensación de rojo, es un objeto, y que, si bien tener el concepto de un objeto no requiere tener una percepción del mismo, tener el concepto de una sensación sí requiere tener la sensación en cuestión. Considero que esto no es sino un prejuicio empirista restringido al ámbito de los conceptos de estados mentales, es decir, a los conceptos psicológicos. Alternativamente, la razón por la cual puede sostenerse que para tener el concepto de una sensación es necesario

21 De ahí que pueda decirse, volviendo al ejemplo de Jackson, que Maria, si bien nunca tuvo una experiencia visual del color, ciertamente tiene el concepto de una experiencia tal.

22 Levin (1986), p. 489. 
tener la sensación en cuestión es que hay aspectos (los qualia) de la sensación que sólo pueden ser captados teniendo la sensación (y no teniendo el concepto). Pero esto es justamente lo que se quiere probar: no es posible argumentar en favor de la existencia de los qualia apelando a la diferencia entre tener el concepto de un objeto y tener el concepto de una sensación sin caer en un círculo. En otras palabras, o bien se provee de una razón independiente de la existencia de los qualia para creer que tener el concepto de una sensación requiere tener la sensación misma (aun cuando ése no sea el caso para los conceptos de objetos) o bien, como creo, no hay razón alguna para mantener una concepción empirista de los conceptos psicológicos.

Para decirlo en términos levemente distintos, puede pensarse que las adscripciones de percepciones involucran dos maneras distintas de relacionarse con un mismo contenido. Mientras que en las adscripciones de creencias el intérprete y el agente tienen ambos el mismo tipo de relación ${ }^{23}$ con cierto contenido, en las adscripciones de percepciones el intérprete no necesita tener con el contenido adscrito una relación en absoluto similar a la que se adscribe al agente. A modo de ejemplo, una persona no necesita ver una manzana roja a fin de adscribirle a otra la percepción visual de una manzana roja - puede inferir tanto el color como el objeto vistos a partir de otros datos, siendo incluso ciega al color. Considérese la siguiente emisión de María, realizada desde fuera del cuarto en el que están Juan, una mesa y una manzana:

(AP) Juan ve que la manzana que está sobre la mesa es roja.

La cláusula subordinada "la manzana que está sobre la mesa es roja" especifica el contenido tanto (de parte) del pensamiento de María como (de la totalidad) de la visión de Juan. ${ }^{24}$ En otras palabras, Juan se representa el contenido de su experiencia bajo la relación de ver — por familiaridad-; María se representa el contenido de la experiencia de Juan bajo la relación de pensar - por descripción. Más aún, si María entrara a la habitación, sería capaz de mantener una relación perceptual con la manzana roja similar a la que mantiene Juan. Si las adscripciones perceptuales pueden ser entendidas de esta manera, entonces una diferencia en la relación con el contenido (como es la diferencia entre tener el concepto de la sensación de rojo y, por tanto, de rojo, por un lado, y tener la sensación de rojo, por otro)

23 Me refiero a una relación teórica. Por supuesto, no necesito creer que la Tierra es plana para atribuir tal creencia a los etruscos; pero debo tener algún tipo de relación teórica o proposicional con el contenido adscrito puesto que debo comprenderlo (a menos que se piense que podemos adscribir contenidos que no comprendemos).

24 En este punto se presupone la tesis, común a muchos funcionalistas, de que el contenido de las representaciones mentales es equivalente al contenido $\multimap 0$ significado- de las representaciones lingüisticas que las expresan. 
no será suficiente para fundamentar una diferencia de contenido (como la supuesta entre el contenido de la sensación conceptualizada y el contenido de la sensación experimentada).

Si esto es así, el que no podamos tener la experiencia de otro (por ejemplo, la de ecolocarse) no implica que no podamos saber en qué consiste tener esa experiencia. Por un lado, como hemos visto, es posible pensar que podemos conocer el contenido de la experiencia de otro modo, es decir, de un modo no experiencial. Por otro, cabe pensar además que podemos conocer, si bien no por tenerla, en qué consiste tener la experiencia, i.e., podemos ser capaces de especificar completamente la experiencia. Tomando otro ejemplo, yo no he estado en la luna por lo que no he tenido la experiencia de ver mis pies sobre la luna; sin embargo, esto no implica que no sea capaz de comprender plenamente el contenido de "Veo mis pies sobre la luna" o de especificar en qué consiste tener esa experiencia visual, por ejemplo, diciendo que consiste en ser el sujeto (en primera persona) de la misma. ${ }^{25}$ De acuerdo con esto, ni la especificación del contenido de la experiencia ni la de la experiencia misma involucran la introducción de componentes esencialmente subjetivos, derivados de cómo es ("what it is like") tener la experiencia.

La interpretación de dicto. Ahora bien, podría considerarse que el verbo "conocer" o "saber" introduce un contexto opaco u oblicuo, en donde los contenidos de las representaciones mentales involucradas no están constituidos por los objetos denotados sino por los sentidos expresados por las correspondientes expresiones del lenguaje natural. Frege, por ejemplo, sostiene que en los contextos gobernados por verbos de actitudes proposicionales las expresiones no denotan su referencia sino su sentido habitual. ${ }^{26}$ En este punto, cabe aclarar que no pretendo comprometerme con ninguna explicación particular de qué sean los sentidos sino tan sólo con la idea fregeana general de que se trata de modos de presentación de los referentes. El punto es que de premisas acerca de modos de presentación de los objetos no es posible derivar una conclusión acerca de los objetos mismos sin cometer una falacia —esta vez, la llamada "falacia intensional". ${ }^{27} 28$ Compárese nuestro ejemplo principal con el siguiente:

$\left(1^{\prime \prime \prime}\right)$ María sabe que la estrella matutina es $F$ (donde $F$ es un predicado complejo que contiene todos los predicados verdaderos de la estre-

25 Véase Lormand (1995b), pp. 17-18, para un desarrollo similar aplicado a conceptos.

26 Véase Frege (1879).

27 Véase Churchland (1985), pp. 19-20.

28 Cabe destacar que los sentidos o modos de presentación y los referentes constituyen distintos tipos de contenidos que pueden adscribirse tanto a las representaciones mentales como a las representaciones del lenguaje natural que las expresan. 
lla matutina con excepción del predicado "ser idéntico a la estrella vespertina"). ${ }^{29}$

$\left(2^{\prime \prime \prime}\right)$ María no sabe que la estrella vespertina es $F$.

Luego, ( $\left.3^{\prime \prime \prime}\right)$ la estrella vespertina es distinta de la estrella matutina.

Bajo una interpretación de dicto de las premisas, María puede saberlo todo acerca de la estrella matutina pero ello sólo incluye las características del objeto bajo cierto modo de presentación del mismo, como la estrella matutina: no incluye el hecho de que brilla durante la noche. En otras palabras, el contenido de la representación mental [la estrella matutina] no es el referente - (Venus) - sino un modo de presentación del mismo - <la estrella matutina). ${ }^{30}$ La conclusión no se sigue: la estrella matutina puede ser la estrella vespertina aun cuando María ignore que son idénticas. Del mismo modo, conocer todos los hechos físicos acerca de la visión del color implica conocer todas las características del fenómeno bajo cierto modo de presentación del mismo, a saber, como visión física del color: no implica conocerlo como experiencia visual. En otras palabras, puede considerarse que el contenido de [visión física del color] no es un fenómeno sino cierto modo de presentación del mismo - (visión física del color . Nuevamente, la conclusión no se sigue: la experiencia visual podría ser enteramente física aun cuando María ignorara el sentido de una manera no física (en el sentido de no propia de la ciencia física) de hacer referencia a ella.

En otras palabras, bajo una interpretación de dicto de las premisas, el argumento es falaz porque una diferencia en el sentido captado (que determina una diferencia en el concepto representado) no puede fundamentar una diferencia en el objeto externo. El argumento sólo podría ser considerado válido bajo el presupuesto de que la realidad está determinada por nuestras maneras de hacer referencia a ella. De lo contrario, mostrar que hay más de una manera de referirse a un objeto no implica mostrar que hay más de un objeto referido.

Como conclusión, considero que el argumento, tanto en su interpretación de re como en su interpretación de dicto, es inválido, a menos que se sostenga alguna versión del antirrealismo metafísico, es decir, una teoría según la cual la epistemología (en la interpretación de re) y la semántica (en la interpretación de dicto) determinan la metafísica; una concepción según la cual nuestro conocimiento del mundo se basa en nuestro conocimiento de nuestro conocimiento y nuestro lenguaje; en tal caso, los objetos existentes

29 Debo esta formulación de las premisas al evaluador de Crítica.

30 Cabe recordar que los corchetes comunes son la convención elegida para designar representaciones mentales y los corchetes angulares son la convención elegida para designar contenidos (tanto referentes como sentidos). 
dependerán de nuestras habilidades subjetivas para conocerlos o referirnos a ellos. ${ }^{31}$ En mi opinión, la estrategia del argumento epistemológico, consistente en fundar la existencia de una propiedad que es un aspecto del contenido (el quale) en nuestra manera (ya sea epistémica o semántica) de acceder a ella (la experiencia o el particular modo de presentación del objeto referido, respectivamente), sólo podría funcionar sobre la base del mencionado presupuesto. Si esto es correcto, entonces el argumento epistemológico no tiene fuerza por sí mismo, es decir, independientemente de un argumento más general en favor de una metafísica antirrealista. En otras palabras, mostrar que tener un conocimiento teórico de algo es distinto de tener una experiencia perceptual no es suficiente para mostrar que lo conceptualizado y lo percibido son ontológicamente distintos. Asimismo, mostrar que acceder a un objeto bajo cierto modo de presentación del mismo es distinto de acceder a él bajo cualquier otro modo de presentación no es suficiente para mostrar que el objeto presentado es en cada caso distinto. ${ }^{32}$

\section{Un problema general}

Los antes mencionados argumentos en favor de los qualia pueden esquematizarse de la siguiente manera:

(1a) argumento epistemológico: Si uno conoce $F$ sin conocer $Q$, entonces $Q$ es distinto de $F$;

(1b) argumento basado en la perspectiva: $Q$ es subjetivo (o puede ser conocido desde la perspectiva de la primera persona) pero $F$ es objetivo (o puede ser conocido desde la perspectiva de la tercera persona), entonces $Q$ es distinto de $F$;

(2a) argumentos basados en la concebibilidad (argumento del Espectro Invertido y argumento de la Tierra Invertida): si uno puede concebir $Q$ sin concebir $F$ y viceversa, entonces $Q$ es distinto de $F$;

31 Vale la pena destacar que es la epistemología empirista la que condujo en última instancia al antirrealismo metafísico - como en Berkeley y los positivistas lógicos. Esto concuerda con el comentario anterior según el cual un defensor de los qualia estaría comprometido con una concepción empirista de (por lo menos) los conceptos psicológicos.

32 Esto no debe inducir a la idea de que intento adscribir tal metafísica antirrealista a defensores de la existencia de los qualia como Nagel y Jackson; por el contrario, mi crítica a ellos es que sus argumentos en favor de los qualia presuponen premisas antirrealistas que muy probablemente ninguno de ellos quiera defender o que están muy alejadas de sus explícitas aspiraciones teóricas. (Debo la inserción de esta aclaración a una observación del evaluador de Diánoia.) 
(2b) argumento basado en la posibilidad: si es posible o concebible como real que $F$ sea distinto de $Q$, entonces $F$ es distinto de $Q .{ }^{33}$

El motivo de especificar tales esquemas es que hacen vívida una característica común: todos van de premisas epistémicas (enunciados acerca de de qué tipo de conocimiento se trata, qué punto de vista puede adoptarse, qué puede concebirse) ${ }^{34}$ a una conclusión ontológica (los qualia existen). De ahí que considere que la estrategia epistémica es, en definitiva, común a todos los argumentos presentados. Más específicamente, se sostiene que los qualia existen porque (1a) tener un concepto de un estado mental es distinto de percibirlo o experimentarlo; (1b) los estados mentales aparecen desde la primera persona de manera distinta de como lo hacen desde la tercera persona; (2a) podemos concebir estados mentales que no son estados funcionales; $y$ viceversa $(2 \mathrm{~b})$ podemos concebir estados funcionales que no son estados mentales. En cada caso, se considera que los qualia son (1a) lo que sólo puede conocerse por medio de la experiencia (en términos de Russell, por familiaridad), (1b) lo que sólo puede conocerse desde la primera persona, (2a) lo que puede concebirse como un aspecto no funcional de la mente y (2b) lo que permite distinguir un estado funcional mental de uno no mental. ${ }^{35}$

Sin embargo, tener un concepto de un estado mental puede ser distinto de experimentar un estado mental pero esto no implica que el estado mental conceptualizado sea distinto del estado mental experimentado. Un estado mental puede aparecer de distinta manera desde el punto de vista de la primera persona de como lo hace desde el punto de vista de la tercera persona, pero eso no implica que sea en cada caso un estado diferente. Asimismo, podemos concebir aspectos mentales que no son funcionales pero eso no implica que esos aspectos existan. Finalmente, podemos concebir estados funcionales que no son estados mentales pero eso no implica que tales estados existan. ${ }^{36}$ En general, a menos que creamos que la epistemología determina la metafísica -0 , en otros términos, que nuestras categorías metafísicas dependen de nuestras categorías epistémicas, a la manera de los empiristas y los fenomenalistas- nuestros conceptos de $F$ y $Q$, nuestro

33 Cabe recordar que la clasificación de los argumentos en dos grandes grupos, (1) y (2), se basa en el énfasis dado al carácter inefable e intrínseco de los qualia respectivamente (véase pp. 3 y 4 ).

34 Obsérvese que todas las premisas contienen términos epistémicos.

35 Nótese, nuevamente, que los verbos destacados con itálicas involucran una actividad epistémica.

36 Esto puede inscribirse en un marco general de desconfianza respecto de la pertinencia, para la teoría de los contenidos mentales, de los argumentos basados en intuiciones modales. 
conocimiento acerca de $F$ y de $Q$ puede ser diferente sin que por ello $F$ y $Q$ sean diferentes.

Como conclusión, en la medida en que los argumentos mencionados involucran una estrategia epistémica, considero que no ofrecen razones suficientes para creer que la experiencia tenga propiedades privadas e inefables. La existencia de tales propiedades requiere como fondo una concepción de la relación entre el conocimiento y el lenguaje, por un lado, y la realidad, por el otro, según la cual los primeros determinan la segunda. Pero esta concepción (antirrealista) ${ }^{37}$ requiere, por supuesto, un argumento independiente.

Dejando de lado esta objeción general, me centraré ahora en los argumentos basados en la concebibilidad, el argumento del Espectro Invertido y el de la Tierra Invertida, los cuales se centran en el carácter intrínseco o no relacional de los qualia. En mi opinión, los argumentos en cuestión son también, y fundamentalmente, vulnerables a objeciones más específicas.

\section{Crítica de la estrategia de la intrinsecalidad}

\section{El Espectro Invertido}

La versión más antigua del argumento del Espectro Invertido (en adelante, AEI) se remonta a Locke. ${ }^{38}$ Hay dos versiones contemporáneas: la intersubjetiva y la intrasubjetiva. ${ }^{39}$ En el primer caso, se nos propone imaginar a un par de gemelas, a una de las cuales se le implantan, al nacer, lentes invertidoras del color. $\mathrm{Al}$ crecer, el contenido intencional de sus representaciones de color es igual al de las representaciones de su hermana -dado que ambas aprenden el significado de los términos de color a partir de su relación con los objetos del compartido mundo externo-, pero sus experiencias visuales son muy distintas - debido a las lentes invertidoras en una de ellas. Se concluye entonces que el contenido de la experiencia visual ha de tener un componente no intencional que explique la diferencia entre las funcionalmente idénticas gemelas: a saber, el componente cualitativo, constituido, en cada caso, por el correspondiente quale.

La versión intrasubjetiva de este experimento mental tiene la ventaja de eludir toda comparación interpersonal. Esta vez, se trata de imaginar a una persona cualquiera a quien se le implantan lentes invertidoras del color mientras duerme. Cuando despierta, el pasto se le aparece rojo y la sangre verde, de modo tal que emite "rojo" delante del pasto y "verde" delante

37 Se presupone aquí una concepción más o menos clásica del realismo, según la cual se trata de una postura que considera que la realidad existe de manera objetiva e independiente de lo mental.

38 Locke (1689) (1980), Libro Il, cap. xxxii, §15.

39 Véase n. 14. 
de la sangre, pero recuerda mientras tanto que ambos solían aparecérsele de distinto color. Después de un tiempo, la persona comienza a aplicar "verde" al pasto y "rojo" a la sangre a pesar del modo en que se le aparecen. Finalmente, pierde todo recuerdo de la época previa a la operación. Esta versión intenta probar que una persona puede sufrir un cambio mental sin cambiar sus estados funcionales. Se concluye, nuevamente, que las representaciones mentales tienen algo más que contenido intencional, a saber, contenido cualitativo.

Las distintas etapas del experimento pueden sintetizarse en el cuadro siguiente:

Objeto/Color Input Contenido Quale Output Memoria

Etapa 1: experiencia visual de una persona normal

\begin{tabular}{|c|c|c|c|}
\hline $\begin{array}{l}\text { pasto verde } \\
\text { sangre roja }\end{array}$ & $\begin{array}{l}\text { verde } \\
\text { roja }\end{array}$ & $\begin{array}{l}\text { 〈verde } \\
\langle\text { roja }\end{array}$ & $\begin{array}{l}\text { verde } \\
\text { roja }^{\prime}\end{array}$ \\
\hline
\end{tabular}

Etapa 2: experiencia visual de una persona con lentes invertidoras

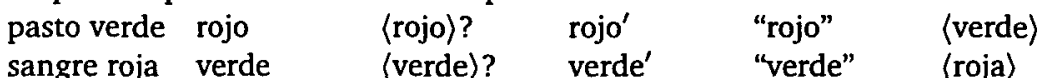

Etapa 3: idem. 2, con adaptación funcional parcial pasto verde rojo $\quad$ rojo)? rojo' "verde" 〈verde sangre roja verde 〈verde)? verde' "roja" 〈roja〉

Etapa 4: idem. 2, con adaptación funcional total

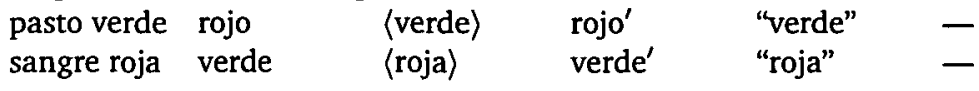

Una primera lectura del cuadro permite afirmar que, en el caso normal (etapa 1), hay una covariación sistemática entre la propiedad objetiva, ${ }^{40} \mathrm{el}$ input subjetivo, el contenido, el quale y el output. Denominaré esta covariación sistemática característica de la etapa 1 "cadena funcional normal". En la etapa 2, es la relación normal entre el mundo y el input lo que es suspendido por la inserción de las lentes invertidoras, mientras que las otras relaciones permanecen inalteradas. Parecería entonces que la cadena funcional pasara a depender del input: frente al pasto, la persona recibe un input rojo, de modo que forma el contenido $\langle$ rojo $\rangle$ o, según algunas versiones del experimento, ningún contenido en particular dado el estado de confusión-, el quale rojo' y dice "rojo". En la etapa 3, es la última parte de la cadena lo que varía: la persona empieza a llamar "verde" a lo que se representa rojo -0 , nuevamente, de ninguna manera en particular, dada la confusión del momento- y se le aparece rojo. Esta etapa ha sido llamada

40 Con el fin de simplificar, daré por sentado que los colores son propiedades intrínsecas de los objetos - pace Locke. 
"adaptación funcional parcial" porque parte de la cadena funcional normal (a saber, el output) ha sido reestablecida. Pero, ¿cómo es posible, dado el input invertido? La adaptación parece ser el resultado de un esfuerzo por superar un input inusual, puesto que la persona se da cuenta perfectamente de que no lo comparte con los demás. ${ }^{41}$ Finalmente, en la etapa 4 , se produce la adaptación funcional total: el punto del argumento consiste en adscribir a esta etapa una recuperación del contenido intencional característico de la etapa 1, a saber, (verde) (ante el pasto). Se sostiene que, desde el punto de vista funcional, no hay diferencias entre las etapas 1 y 4; sin embargo, los estados mentales correspondientes son distintos en otro aspecto, a saber, son cualitativamente distintos; por consiguiente, los estados mentales no pueden ser identificados con -0 reducidos a- estados funcionales.

Ahora bien, mi objeción fundamental es que ningún criterio de individuación del contenido puede servir como base para fundamentar lo que el argumento se propone, a saber, la idea de que en 4 hay una recuperación del contenido intencional original -0 , en otros términos, la idea de que no hay diferencia intencional alguna entre 1 y 4 . En lo que sigue, intentaré fundamentar esta objeción.

En mi opinión, la pregunta clave es isobre qué fundamentos puede adscribirse una reinversión intencional en la etapa 4? Una, o más de una, de las siguientes razones, podrían alegarse:

(i) el objeto referido (a saber, el pasto) no es rojo (criterio externalista)

(ii) la persona dice "verde" (no dice "rojo") (criterio internalista)

(iii) cierto recuerdo conflictivo ( $\langle$ verde $\rangle$ ) ha desaparecido (criterio internalista).

Cabe aclarar que los criterios externalistas son los propuestos por las teorías externalistas del contenido intencional, es decir, aquéllas según las cuales el contenido de las representaciones mentales está constituido por las relaciones entre éstas y los objetos del mundo externo; por otro lado, los teorias internalistas defienden criterios internalistas según los cuales el contenido de las representaciones mentales está constituido por sus relaciones internas con otras representaciones. ${ }^{42}$ Ahora bien, si (i) es ignorado en la etapa 3, ¿por qué habría de ser considerado en 4 ? Los fundamentos para ignorar el objeto en las etapas 2 y 3 (es decir, el hecho de que se pierde

41 En este aspecto, el caso descrito difiere radicalmente de cualquier caso de daltonismo, donde la persona suele ser completamente inconsciente de la diferencia entre sus contenidos visuales y los de los demás.

42 Como ejemplo de las primeras cabe mencionar la teoría histórico-causal de la referencia presentada en Kripke (1981); como ejemplo de las segundas, la teoría del rol conceptual propuesta en Block (1986). 
la conexión causal normal) son fundamentos para ignorarlo en la etapa 4. En lo que se refiere a (ii), la persona puede conducirse de ese modo con el único objeto de adaptarse a su comunidad lingüística. A menos que se adhiera al conductismo, es posible creer que la persona continúa con una farsa iniciada en la etapa 3: sigue llamando "verde" a lo que se representa y se le aparece rojo de modo tal de ajustar su conducta lingüística a los significados públicos.

El punto (iii) indica, en realidad, la única diferencia entre las etapas 3 y 4. Por consiguiente, la adscripción del cambio intencional entre ambas etapas (es decir, la vuelta a 1 en la etapa 4) está basada en un criterio internalista. Sin embargo, en primer lugar, no puede tratarse de un criterio basado en el rol conceptual. Desde este punto de vista, el contenido en la etapa 4 es claramente distinto del contenido en la etapa 1 puesto que cada caso involucra distintas propiedades inferenciales: en la etapa 1, 〈verde〉 está

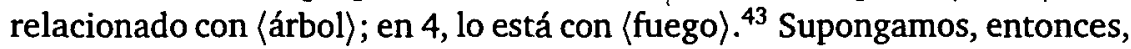
que el contenido de una representación-caso es individuado en términos del contenido de una representación-tipo, es decir, en términos de las interacciones causales típicas con inputs, outputs y otras representaciones (lo que he estado llamando "cadena funcional normal"). ¿Cómo es posible que la desaparición de cierto recuerdo - 〈el pasto solía lucir verde $\rangle$ - haga variar el contenido de 〈rojo〉 a 〈verde〉? Más específicamente, ¿cómo es posible que el olvido por parte del sujeto del hecho de que el pasto solía lucir verde constituya un fundamento teórico para adscribirle el contenido 〈verde) a su sensación y a su consiguiente emisión de "verde"? 44

Sobre la base de lo anterior, mi diagnóstico es el siguiente: en la etapa 2 se produce una inversión intencional respecto de la etapa 1 y posteriormente no hay reinversión intencional alguna -ni en 3 ni en 4 . A continuación, explicitaré cuál es el criterio que considero que debe aplicarse para la individuación de los contenidos perceptuales y describiré cómo puede entenderse cada etapa del experimento a la luz de aquél.

En primer lugar, creo que el criterio adecuado para la individuación de los contenidos perceptuales es un criterio (externalista pero no histórico sino) indicativo-causal: de acuerdo con éste, un sujeto se representa un

43 En última instancia, de acuerdo con el holismo extremo de algunas teorias de roles conceptuales, tanto 〈árbol) como 〈fuego〉 son parte del contenido de [verde] -y de "verde"en ambas etapas. Pero si esto probara algo, probaría demasiado: probaría que los contenidos de todos los conceptos son idénticos, en tanto constituidos por la misma totalidad de propiedades inferenciales.

44 Paradojicamente, mientras el recuerdo (verde) está presente, el contenido en cuestión no es, con certeza, (verde); cuando el recuerdo desaparece, el contenido pasa a ser, con certeza, (verde). 
objeto como verde si tiene una relación causal con el color verde, la cual está basada en las capacidades discriminatorias comunes a la especie. ${ }^{45}$ En general, esto involucra un input verde, un ouput "verde" y un recuerdo 〈verde), sin ser éstos últimos los que determinan el contenido sino la relación causal en cuestión. Ahora bien, las lentes invertidoras modifican la relación causal: en virtud de aquéllas, tener una relación causal con algo verde conduce a representárselo rojo, lo que entra a la conciencia es el contenido 〈rojo). Como se describe en la etapa 2, se tiene entonces un input rojo y un output "rojo". Como dije antes, la cadena funcional empieza a depender del input. Esto puede conducir a creer que el contenido es individuado con un criterio internalista; mi diagnóstico, sin embargo, es que lo que determina el contenido es una relación causal inusualmente compleja, en tanto incluye un par de lentes invertidoras. Más aún, pienso que es el hecho de que el sujeto es consciente de un problema de comunicación lo que lo conduce a usar, en la etapa 3, la palabra "verde" para lo que se representa - no confusa sino claramente- rojo, ignorando de esta manera la relación

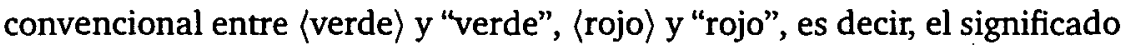
de los términos de color. En la etapa 4, acaba por olvidar completamente esa convención: empieza a hablar otro lenguaje de color, una suerte de lenguaje privado, donde "verde" significa 〈rojo〉 y "rojo" significa 〈verde $\rangle.$ Según esto, en la etapa 4, la adaptación de la conducta no involucra una reinversión intencional sino la adquisición de un nuevo lenguaje de color que mantiene el contenido intencional tan invertido como al presunto contenido cualitativo (que, de este modo, no tiene por qué ser concebido como un factor independiente y distinto del contenido intencional).

Tal vez pueda objetarse que esta explicación evita los qualia a un costo muy alto: se pierden las conexiones regulares entre inputs, contenidos y conductas. Pero, iestamos en posición de exigir tales regularidades en casos tan atípicos como el del experimento? Y itiene esto alguna consecuencia para los casos normales?

Finalmente, quiero mencionar dos razones por las que encuentro esta hipótesis aceptable. En primer lugar, respeta la conexión input-contenido: el contenido 〈verde) exige un input verde. ${ }^{46}$ De acuerdo con el modo en que el experimento es descrito, no hay razón para creer que esa conexión deba ser modificada por el uso de lentes invertidoras. Más específicamente, sin presuponer una inversión quirúrgica en otros puntos de la cadena funcional, no tiene sentido afirmar que el sujeto ve verde cuando recibe un estímulo rojo, como sugiere la descripción de la etapa 4 por parte de los defensores de AEI. Por otro lado, la hipótesis sugerida respeta la conexión contenido-quale: el

45 Véase la teoría de Dretske en Dretske (1981).

46 Cabe preguntarse, por supuesto, qué son, exactamente, los inputs. 
contenido 〈verde) está relacionado sistemáticamente con un quale verde ${ }^{\prime}{ }^{47}$ Si hay qualia (y éstos son las condiciones de posibilidad de la conciencia, es decir, son lo que hace, para seguir con el ejemplo, a las sensaciones de verde sensaciones conscientes), no pueden estar relacionados con los contenidos intencionales de la manera en que lo sugiere la descripción oficial de la etapa 4: ¿qué podría querer decir que alguien tiene una sensación de verde que se le aparece como una sensación de rojo? Peor aún, ¿cómo es posible que lo que otorgue carácter consciente a su sensación de verde sea el ser sentida ("feels like") como una sensación de rojo? Nuevamente, si no se presupone una inversión quirúrgica en otros puntos de la cadena funcional, en particular, entre la representación del contenido intencional y la representación de las propiedades cualitativas, no tiene sentido afirmar que el sujeto se representa algo verde que es sentido como rojo. Esta última consideración muestra claramente que no pretendo argumentar en contra de los qualia en general sino en contra de los argumentos ofrecidos en su favor como propiedades intrínsecas de la experiencia.

Como conclusión, si no hay reinversión intencional en la etapa 4 , o, en otras palabras, si hay una diferencia intencional entre las etapas 1 y 4 , la diferencia entre ambas etapas puede ser explicada en términos intencionales. Si esto es así, el Espectro Invertido no constituye un argumento en favor de la existencia de un aspecto no intencional de los contenidos de la conciencia.

\section{La Tierra Invertida}

En un artículo comparativamente reciente, Block ha ofrecido un nuevo argumento en favor de la existencia de un aspecto no intencional de la mente basado en el experimento mental de la Tierra Invertida (en adelante, ATI). ${ }^{48}$ A continuación, sintetizaré brevemente su exposición de este experimento. Block nos incita a imaginar que una de dos hermanas gemelas recibe un par de lentes invertidoras, luego de lo cual, en lugar de permanecer en la Tierra junto a su hermana, es enviada a la Tierra Invertida. Dos tipos de inversión sirven para caracterizar este fantástico planeta: en primer lugar, los colores están invertidos respecto de los colores de la Tierra (el pasto es rojo, la sangre es verde, el cielo es amarillo, el sol es azul, etc.); en segundo lugar, los significados de los términos de color están también invertidos respecto de los significados terrenos (en el lenguaje Invertido, "rojo" significa 〈verde〉, "verde" significa 〈rojo); "amarillo" significa 〈azul〉, "azul"

47 La sistematicidad de la relación en cuestión es de carácter intuitivo: no presupone ninguna teoría particular acerca de los qualia o de la conciencia sino que toda teoría particular al respecto deberá dar cuenta de ella.

48 Véase n. 15. 
significa (amarillo〉, etc.). Ambas inversiones tienen como consecuencia la cancelación del efecto de las lentes invertidoras de modo tal que, durante cierto periodo de tiempo, la experiencia y la conducta de la gemela en la Tierra Invertida serán idénticas a la experiencia y la conducta de su hermana en la Tierra. A modo de ejemplo, frente a los respectivos mares, ambas tendrán una sensación de azul y dirán "mar azul", haciendo referencia, en ambos casos, al azul mar terreno. Pero, cincuenta años más tarde, la gemela enviada a la Tierra Invertida habrá empezado a hablar el lenguaje de la Tierra Invertida, por lo que su emisión "mar azul" no hará ya referencia al azul mar terreno sino al mar amarillo de la Tierra Invertida. En ese entonces, aun cuando sus respectivas experiencias continúen siendo idénticas respecto de su carácter cualitativo (ambas tendrán un quale azul'), diferirán en el objeto referido (〈el azul mar de la Tierra) y (el mar amarillo de la Tierra Invertida), respectivamente), es decir, en su contenido intencional.

Como puede imaginarse, el experimento puede ser reformulado para el caso intrasubjetivo, donde se trata de comparar las sucesivas experiencias o momentos experienciales de una misma persona - antes y después de recibir las lentes invertidoras y ser enviada a la Tierra Invertida. De este modo, se llegará a la misma conclusión: después de haber vivido en la Tierra Invertida durante cincuenta años, el factor intencional del contenido de la experiencia habrá cambiado, sin variación cualitativa concomitante.

Las distintas etapas pueden ser sintetizadas en un cuadro de la siguiente manera:

Objeto/Color Input Contenido Quale Output

1. Experiencia visual de un nativo de la Tierra

\begin{tabular}{|c|c|c|}
\hline $\begin{array}{l}\text { narillo } \\
\text { azul }\end{array}$ & $\begin{array}{l}\text { amarillo } \\
\text { azul }\end{array}$ & $\begin{array}{l}\text { (amarillo }\rangle \\
\langle\text { azul }\rangle\end{array}$ \\
\hline
\end{tabular}

2. Experiencia visual de un nativo de la Tierra Invertida

$\begin{array}{lllll}\text { sol I azul } & \text { azul } & \text { (azul〉 } & \text { azul' } & \text { "amarillo" en LI } \\ \text { cielo I amarillo amarillo } & \text { (amarillo) } & \text { amarillo" } & \text { "azul" en LI }\end{array}$

3. Experiencia visual de un nativo de la Tierra enviado a la Tierra Invertida con lentes invertidoras

$\begin{array}{lllll}\text { sol I azul } & \text { amarillo } & \text { (amarillo) } & \text { amarillo' } & \begin{array}{l}\text { "amarillo" en L } \\ \text { cielo I amarillo }\end{array} \\ \text { azul } & \text { (azul) } & \text { azul' } & \text { azul" en L }\end{array}$

4. Idem. 3, después de cincuenta años

\begin{tabular}{|c|c|c|}
\hline ul & $\begin{array}{l}\text { amarillo } \\
\text { azul }\end{array}$ & $\begin{array}{l}\langle\text { azul }\rangle \\
\langle\text { amarillo }\rangle\end{array}$ \\
\hline
\end{tabular}

Así como el anterior, este nuevo experimento mental se propone mostrar que hay dos tipos de contenido: el intencional y el cualitativo. Se supone 
entonces que constituyen dos aspectos diferentes de la mente: el aspecto cualitativo no puede ser reducido a $\longrightarrow$ identificado con- el aspecto funcional.

Ahora bien, en mi opinión, la clave de ATI reside en la tesis de que en la etapa 4 hay un cambio intencional respecto de 3 , la cual se fundamenta en la adscripción de un cambio de lenguaje. Pero, en mi opinión, esta última afirmación depende de una dudosa concepción del contenido de las representaciones perceptuales. En lo que sigue, desarrollaré primero esta objeción para luego proponer una concepción alternativa que hace posible una interpretación distinta del experimento.

Lo que conduce a pensar que en la etapa 4 hay un cambio de lenguaje respecto de 3 es claramente una concepción externalista históricocausal del significado de los términos perceptuales. En 3, "amarillo" significa 〈amarillo) porque está histórico-causalmente fundada en el color amarillo; en 4 "amarillo" significa 〈azul〉 por una razón paralela. En ningún caso se considera que el modo en que el color en cuestión se le aparece al sujeto forma parte del significado intencional; lejos de ello, se considera que constituye un aspecto independiente, de tipo cualitativo. Por lo tanto, según Block, para explicar el contenido total de las representaciones perceptuales, es necesario contar con dos teorías distintas. Por un lado, se requiere una teoría histórico-causal de la referencia, según la cual la referencia de un término perceptual es identificada con el objeto/propiedad con el/la cual el término se halla histórico-causalmente relacionado -semejante a la teoría propuesta por Kripke para los nombres propios y términos generales. ${ }^{49}$ Por otro, se requiere una teoría no funcionalista del modo de presentación del objeto/propiedad en cuestión, modo que se considera, no una parte del contenido intencional de la representación mental asociada sino un contenido cualitativo independiente, es decir, un contenido que no puede ser explicado en términos funcionales.

Ahora bien, por un lado, si se sostiene junto con Block que los qualia son contenidos cualitativos independientes, nuevamente, se nos deberá una explicación de cómo es posible para la gemela con lentes invertidoras tener una consciente sensación de azul por medio de un quale amarillo' (etapa 4). Si los qualia son aquello que hace posible la conciencia (véase introducción) entonces ¿cómo puede ser que un quale amarillo' determine el carácter consciente de una sensación de azul? Dado que no hay inversiones posinput, eso no parece tener sentido alguno. La única manera de dar algún sentido a tal afirmación es creer que el quale amarillo' determina el carácter consciente de una sensación que es internamente una representación de amarillo, si

49 Véase Kripke (1981). 
bien está externamente conectada con el color azul. En otras palabras, el quale amarillo' puede considerase correspondiente a un contenido internamente individuado (contenido estricto o "narrow content"). Si esto es así, el experimento no alcanza su propósito fundamental, a saber, distinguir el contenido intencional (estricto) del (supuesto) contenido cualitativo. Lejos de ello, los ítems pertenecientes a la columna de qualia parecen corresponderse sistemáticamente con ítems en una (imaginaria) columna de contenidos (intencionales) estrictos. Pero entonces la razón principal para postular la existencia de los qualia no estará fundada en su carácter intrínseco, no intencional (como pretende el experimento) sino en el hecho de que son necesarios para definir la conciencia. Cabría entonces preguntarse ¿cuál es el verdadero carácter de las propiedades explicativas de la conciencia? El experimento de la Tierra Invertida no proporciona ninguna razón para creer que se trate de propiedades intrínsecas.

Por consiguiente, aun cuando se conceda a Block que la teoría adecuada de los contenidos mentales incluye una teoría histórico-causal de la referencia, tampoco se sigue de ello lo que el experimento pretende probar, a saber, que hay un aspecto del contenido que es puramente cualitativo y no intencional. Como dije antes, el experimento sólo habría probado que es necesario postular, junto al objeto referido, un factor interno del contenido, que recoja el tradicional modo de presentación, i.e., que sea sensible a la discriminación subjetiva. Pero, en ningún caso, se habría probado que tal factor interno haya de tener un carácter no intencional. ${ }^{50}$

En mi opinión, esto muestra que ATI descansa en un injustificado presupuesto: se trata de la tesis de que el objeto referido y el modo de presentación del mismo son de una naturaleza tan distinta que pueden separarse y constituir dos factores independientes. En el caso en estudio, el de las representaciones perceptuales, se presupone que constituyen el contenido intencional, por un lado, y el contenido cualitativo, por otro. Si se tratara de representaciones no perceptuales, se diría que constituyen el contenido amplio y el contenido estricto, respectivamente. Es por ello que considero que el diseño mismo del experimento presupone la llamada "teoría del doble factor", la cual, en tanto aplicada al contenido de los estados mentales llamados "fenoménicos", podría ser denominada "teoría del doble contenido" -por cuanto postula la existencia no ya de dos factores independientes dentro del contenido intencional sino de dos contenidos independientes, el

50 ¿Por qué el modo de presentación del objeto referido habria de constituir un aspecto independiente del contenido, de tipo no intencional? Desde Frege en adelante, los (diversamente concebidos) modos de presentación (o sentidos) han sido considerados partes esenciales del contenido intencional. Si, como sugerl anteriormente, los qualia del experimento pueden ser identificados con los modos de presentación, no hay razón alguna para adscribirles una naturaleza no intencional. 
intencional y el cualitativo. (De hecho, la teoría en cuestión es frecuentemente asociada con el nombre de Block, quien la defiende explícitamente en Block (1986). $)^{51}$ Pero, en mi opinión, no hay razón alguna para creer que existe un profundo divorcio entre el objeto externo referido por una representación perceptual (i.e., la referencia) y el contenido (en tanto sentido o modo de presentación) constituido a partir de él; en otros términos, no hay razón alguna para creer que la teoría del doble factor es verdadera. El experimento de la Tierra Invertida de Block ( $y$ en ello se asemeja al de la Tierra Gemela de Putnam) no proporciona esa razón, sino que la presupone.

Por otro lado, a diferencia de lo propuesto por Block, es posible pensar que el significado de los términos perceptuales depende del contenido de las representaciones mentales asociadas: las condiciones de individuación de éstas últimas serían entonces independientes de la conducta verbal. Podría pensarse que el contenido de tales representaciones depende en cambio del extremo opuesto de la cadena funcional, a saber, del input recibido, el cual, en lo que concierne a las representaciones perceptuales, determina una restricción fundamental en la constitución del contenido. Soy consciente de que la naturaleza de los inputs es vaga y, por lo general, está subdescrita; sin embargo, pienso que pueden ser concebidos (en el caso de las representaciones conscientes) como aquello que entra a la conciencia, ${ }^{52}$ lo cual, para cierto tipo de representaciones mentales como las sensaciones, puede determinar enteramente el contenido. De acuerdo con esto, si se tiene un input azul, no será posible tener una sensación de amarillo, no importa cuán adaptada se muestre la conducta verbal subsiguiente. Por supuesto, podría haber otras restricciones, pero, en lo que concierne a la presente descripción del experimento, podría considerarse que son satisfechas. En otras palabras, si se piensa, como estoy sugiriendo, que (i) el significado de los términos que se refieren a objetos/propiedades perceptuales se deriva del contenido de las representaciones mentales asociadas (y no a la inversa) y (ii) el sujeto del experimento, al recibir, por ejemplo, un input amarillo frente al sol invertido, sólo puede formar la representación [amarillo], entonces no habrá razón para adscribirle un cambio de lenguaje (puesto que la diferencia entre L y LI es sólo la diferencia en el significado de sus respectivos términos de color). Si esto es así, no hay en la etapa 4 una inversión intencional respecto de 3.

En otros términos, como explicité previamente en relación con AEI, considero que una teoría adecuada del contenido de las representaciones percep-

51 Un ejemplo temprano de la teoría es Putnam (1975).

52 Esto parece concordar con lo implicado por los defensores de ATI, por cuanto los qualia, es decir, lo que da a las representaciones su carácter consciente, dependen sistemáticamente de los inputs (véanse las respectivas columnas en el cuadro). 
tuales ha de ser externalista pero de tipo informacional o indicativo-causal - del tipo de la teoría informacional de Dretske o de la teoría covariacional propuesta por Fodor: ${ }^{53}$ de acuerdo con esto, el significado de "amarillo" en 4 está determinado por el contenido de la representación mental asociada, a saber, [amarillo], y este último está constituido por una relación causal con el color amarillo que se basa en la capacidad discriminatoria del emisor; ${ }^{54}$ por lo tanto, el modo de presentación del color en cuestión es parte del contenido intencional. Si esto es así, el significado de "amarillo" emitida por el sujeto en 4 no es - y no puede ser-〈azul〉 sino 〈amarillo); aquello que se instancia en la mente del sujeto es, tanto en la etapa 3 como en la 4, la representación [amarillo]: no hay inversión intencional alguna que contraste con la - supuestamente independiente-continuidad cualitativa.

\section{Conclusión}

Pienso que los argumentos considerados con mayor detalle -el argumento epistemológico, el Espectro Invertido y la Tierra Invertida- son los principales argumentos que han sido esgrimidos en favor de la existencia de los qualia. Ninguno de ellos es, en mi opinión, lo suficientemente persuasivo en favor de la tesis de que los qualia, tal como fueron descritos al comienzo de este artículo, existen. Ahora bien, afirmar que no hay tales qualia no implica afirmar que los estados mentales no tienen componentes cualitativos. Por consiguiente, afirmar que no hay tales qualia no implica no poder trazar la distinción entre conciencia e inconciencia. Como se vio al comienzo, dentro del funcionalismo, ha habido distintos intentos de trazar esta distinción: fundamentalmente, están las teorías metarrepresentacionales (a la Rosenthal) y las teorías caracterizables como estrictamente funcionalistas (a la Dennett). Si bien ninguna de ellas parece ser individualmente exitosa, tal vez una teoría mixta, que combine algún tipo de metarrepresentación con otras relaciones funcionales, lo sea. Tal teoría podría incluir a los qualia como parte de su ontología, pero no ya concebidos como propiedades privadas e intrínsecas sino como propiedades funcionales de ciertos estados mentales. ${ }^{55}$

53 Véase Dretske (1981); Fodor (1990).

54 Soy consciente de que este tipo de teorías están abiertas al llamado "problema del error o la disyunción", a saber, el problema de cómo distinguir las representaciones verdaderas de las falsas, punto que me fue oportunamente señalado por el evaluador de Diánoia. Al respecto, cabe señalar que mi intención en este artículo no es presentar una solución a ese difícil problema sino tan sólo sugerir una línea de investigación en el área de la teoría semántica distinta de la presupuesta por Block - la cual por su parte no deja de presentar problemas igualmente graves y de difícil solución, como el problema del respecto o qua problem.

55 Véase la teoría de Lormand, para quien los qualia son propiedades relacionales de las representaciones perceptuales conscientes, es decir, propiedades que tales representaciones 
En síntesis, mi objetivo en este trabajo no ha sido demostrar que, como quiera que se los conciba, los qualia no existen, sino rechazar algunos argumentos tradicionales en favor de los qualia concebidos como propiedades privadas e inefables (fundamentalmente, el argumento epistemológico) e intrínsecas (argumentos del Espectro y la Tierra Invertidos) de la experiencia. Mi conclusión es que, dado que tales argumentos no son convincentes, no hay razones para creer que tales qualia existan. La conclusión no parece temeraria dado que, si alguna versión no proposicional de la teoría metarrepresentacional o alguna combinación de ésta última con una teoría a la Dennett es exitosa, no es la existencia de tales qualia lo que parece requerirse para la distinción entre conciencia e inconciencia. ${ }^{56}$

\section{REFERENCIAS BIBLIOGRÁFICAS}

Block, N. 1978, "Troubles with Functionalism" en W. Savage (comp.), Perception and Cognition, University of Minnesota Press, Minnesota Studies in the Philosophy of Science, vol. IX, Minneapolis.

-1980, “Are Absent-Qualia Impossible?", Philosophical Review, no. 89.

- 1986, "Advertisement for a Semantics for Psychology" en P. French, T. Uehling y H. Wettstein (comps.), Studies in the Philosophy of Mind, vol. 10 of Midwest Studies in Philosophy, University of Minnesota Press, Minneapolis.

1990. "Inverted Earth" en J. Tomberlin (comp.), Philosophical Perspectives 4: Action Theory and Philosophy of Mind, CA: Ridgeview, Atascadero.

Churchland, P. 1985, "Reduction, Qualia and the Direct Introspection of Brain States", The Journal of Philosophy, LXXXII.

Dennett, D. 1988, “Quining Qualia", en A.J. Marcel y E. Bisiach (comps.), Consciousness in Contemporary Science, The Clarendon Press, Oxford.

-1991, Consciousness Explained, Little Brown, Nueva York.

Descartes, R. 1641, Meditaciones metafísicas, trad. español M. García Morente, Espasa-Calpe, Madrid, 1982.

Devitt, M. 1991, Realism \& Truth, 2a. ed., Blackwell, Cambridge.

Dretske, F. 1981, Knowledge and the Flow of Information, MIT Press, Cambridge, Mass.

tienen en virtud de su relación con percepciones internas que son acerca de ellas. Se trata, por tanto, de una teoría metarrepresentacional, pero la conciencia acerca de la conciencia no es concebida como una conciencia teórica o proposicional, a la Rosenthal, sino como una conciencia perceptual ("inner perception"). Cfr. Lormand (1995a y b).

56 Agradezco a Georges Rey y Eric Lormand las interesantes discusiones que motivaron mi interés por el tema; a Lormand, además, por la lectura y corrección minuciosas de una primera versión de este trabajo; a Eduardo Barrio por sus observaciones críticas y su constante apoyo; finalmente, quiero agradecer al anónimo evaluador de Crítica y Diánoia por los numerosos y lúcidos comentarios y sugerencias que hicieron posible una versión más perspicua del artículo. 
Flanagan, O. 1992, Consciousness Reconsidered, cap. 4, MIT Press, Cambridge, Mass. Fodor, J. 1990, A Theory of Content, MIT (Bradford) Press, Cambridge, Mass.

Frege, G. 1879, "Sobre sentido y referencia", trad. español U. Moulines, en Valdés Villanueva (comp.), La búsqueda del significado, Tecnos, Madrid, 1991.

Harman, G. 1990, "The Intrinsic Quality of Experience", Philosophical Perspectives, no. 4.

Jackson, F. 1982, "Epiphenomenal Qualia", Philosophical Quarterly, no. 32.

Kripke, S. 1981, El nombrar y la necesidad, trad. español M. Valdés, UNAM, México, 1985.

Levin, J. 1986, "Could Love Be a Heatwave? Physicalism and the Subjective Character of Experience", en W. Lycan (comp.), Mind \& Cognition, Blackwell, Cambridge, Mass., 1990.

Levine, J. 1983, "Materialism and Qualia: The Explanatory Gap", Pacific Philosophical Quarterly, no. 64.

Locke, J. 1690, en S. Rabade y Ma. E. García (comps.), Ensayo sobre el entendimiento humano, Editora Nacional, Madrid 1980.

Lormand, E. 1995a, "Qualia! (Now Showing at a Theater Near You)", Philosophical Topics (Dennett issue).

-1995b, "Consciousness", en The Routledge Encyclopedia of Philosophy, Routledge, Londres.

Lycan, W. 1973, "Inverted Spectrum", Ratio, vol. XV.

Nagel, Th. 1974, "What Is It Like To Be a Bat?", Philosophical Review, no. 83.

Putnam, H. 1975, "El significado de 'significado'", trad. español J.J. Acero, en

L. Valdés Villanueva (comp.), La búsqueda del significado, Tecnos, Madrid, 1991.

Rey, G. 1992, "Sensational Sentences Switched", Philosophical Studies, no. 68.

_ 1993, "Sensational Sentences", en M. Davies y G. Humphyries (comps.), Consciousness, Blackwell, Oxford.

Rosenthal, D. 1990. "A Theory of Consciousness", ZIF Report, no. 40, Center for Interdisciplinary Research, Bielefeld.

Shoemaker, S. 1975, "Functionalism and Qualia", Philosophical Studies, no. 27.

-1982, "The Inverted Spectrum", The Journal of Philosophy, no. 79.

-1991, "Qualia and Consciousness", Mind, vol. 4. 